NATIONAL LABORATORY

\title{
Wireless Sensing, Monitoring and Optimization for Campus-Wide Steam Distribution
}

\section{November 2011}

Prepared by

M. M. Olama

G. O. Allgood

P. T. Kuruganti

S. R. Sukumar

K. Woodworth

J. E. Lake

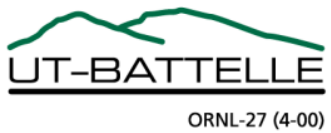




\section{DOCUMENT AVAILABILITY}

Reports produced after January 1, 1996, are generally available free via the U.S. Department of Energy (DOE) Information Bridge.

Web site http://www.osti.gov/bridge

Reports produced before January 1, 1996, may be purchased by members of the public from the following source.

National Technical Information Service
5285 Port Royal Road
Springfield, VA 22161
Telephone 703-605-6000 (1-800-553-6847)
TDD 703-487-4639
Fax 703-605-6900
E-mail info@ntis.gov
Web site http://www.ntis.gov/support/ordernowabout.htm

Reports are available to DOE employees, DOE contractors, Energy Technology Data Exchange (ETDE) representatives, and International Nuclear Information System (INIS) representatives from the following source.

Office of Scientific and Technical Information

P.O. Box 62

Oak Ridge, TN 37831

Telephone 865-576-8401

Fax 865-576-5728

E-mail reports@osti.gov

Web site http://www.osti.gov/contact.html

This report was prepared as an account of work sponsored by an agency of the United States Government. Neither the United States Government nor any agency thereof, nor any of their employees, makes any warranty, express or implied, or assumes any legal liability or responsibility for the accuracy, completeness, or usefulness of any information, apparatus, product, or process disclosed, or represents that its use would not infringe privately owned rights. Reference herein to any specific commercial product, process, or service by trade name, trademark, manufacturer, or otherwise, does not necessarily constitute or imply its endorsement, recommendation, or favoring by the United States Government or any agency thereof. The views and opinions of authors expressed herein do not necessarily state or reflect those of the United States Government or any agency thereof. 
Computational Sciences and Engineering Division

\title{
WIRELESS SENSING, MONITORING AND OPTIMIZATION FOR CAMPUS- WIDE STEAM DISTRIBUTION
}

\author{
M. M. Olama \\ G. O. Allgood \\ P. T. Kuruganti \\ S. R. Sukumar \\ K. Woodworth \\ J. E. Lake
}

Date Published: November 2011

Prepared by

OAK RIDGE NATIONAL LABORATORY

Oak Ridge, Tennessee 37831-6283

managed by

UT-BATTELLE, LLC

for the

U.S. DEPARTMENT OF ENERGY

under contract DE-AC05-00OR22725 



\section{CONTENTS}

\section{Page}

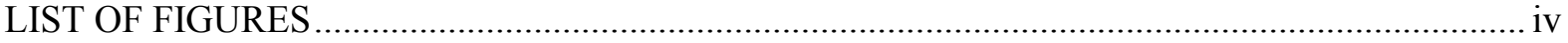

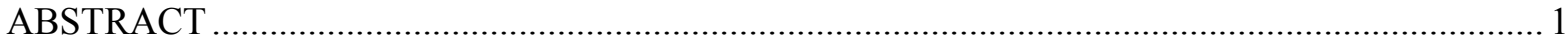

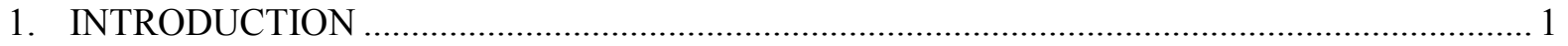

2. COMMUNICATION SYSTEM ARCHITECTURE AND INTEGRATION ............................. 3

3. THE PROPOSED ENERGY SIGNATURE SCHEME ….................................................... 5

4. EXTRACTING SIGNATURES FOR STEAM TRAP CLASSIFICATION …......................... 6

5. EXTRACTING SIGNATURES FOR STEAM FLOW ESTIMATION …................................. 9

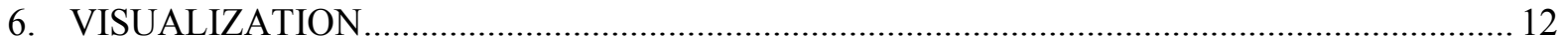

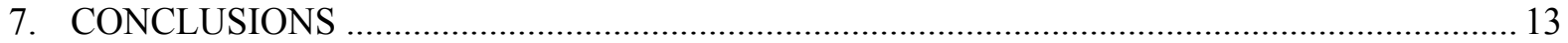

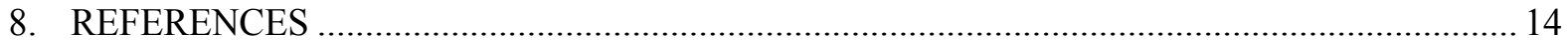




\section{LIST OF FIGURES}

Figure

Page

1 Data-flow architecture of the ORNL wireless energy monitoring system. .3

2 Plan view of ORNL's campus showing the locations of the valves and steam traps in addition to the antenna location and coverage .4

3 Time-frequency PSDs of a single class of steam trap types showing similar operational ranges and varying states.

4

Energy signatures for the three states and health under similar operational conditions (125 psi / 20 lbs.)

5 Energy signature values computed on data acquired from the input and output sections of a steam trap. 8

6 Box-and-whisker plot for 10 data sets collected at a fixed steam flow rate of 2390 Lit/Min and at different time instances over a particular week.

Energy signature values computed on vibration data at different steam flow rates

8 Sensitivity analysis for energy signature values computed on vibration data at different steam flow rates.

The situational awareness interface used to visualize sensor data from the wireless sensors along with status alerts. 


\begin{abstract}
The U.S. Congress has passed legislation dictating that all government agencies establish a plan and process for improving energy efficiencies at their sites. In response to this legislation, Oak Ridge National Laboratory (ORNL) has recently conducted a pilot study to explore the deployment of a wireless sensor system for a real-time measurement-based energy efficiency optimization. For our campus, with particular focus on the 12-mile long steam distribution network, we propose an integrated system-level approach to optimize energy delivery within the steam distribution system. Our approach leverages an integrated wireless sensor and real-time monitoring capability. We make real time state assessment on the steam trap health and steam flow estimate of the distribution system by mounting acoustic sensors on the steam pipes/traps/valves and observing measurements of these sensors with state estimators for system health. Our assessments are based on a spectral-based energy signature scheme that interprets acoustic vibration sensor data to estimate steam flow rates and assess steam traps status. Experimental results show that the energy signature scheme has the potential to identify different steam trap states and it has sufficient sensitivity to estimate flow rate. Moreover, results indicate a nearly quadratic relationship over the test region between the overall energy signature factor and flow rate in the pipe. We are able to present the steam flow and steam trap status, sensor readings, and the assessed alerts as an interactive overlay within a web-based Google Earth geographic platform that enables decision makers to take remedial action. The goal is to achieve significant energy-saving in steam lines by monitoring and acting on leaking steam pipes/traps/valves. We believe our demonstration serves as an instantiation of a platform that extends implementation to include newer modalities to manage water flow, sewage and energy consumption.
\end{abstract}

\title{
1. INTRODUCTION
}

A Department of Energy study published in $2004^{1}$ identified faulty steam traps as a major source of energy waste at industrial sites. The study states that approximately $20 \%$ of the steam leaving a central boiler plant is lost via leaking traps in typical space heating systems. Applying proactive assessment programs, which allow continuous monitoring and evaluation, can reduce losses to less than $1 \%$. The potential impact in the Federal sector is enormous, annual savings associated with implementing a proactive assessment program is estimated to be over $\$ 100$ million.

Our focus in this report is on the high-volume industrial steam distribution systems that deliver steam from a boiler to the end-use operations. Our aim is to present relatively inexpensive sensorbased methods coupled with analysis algorithms for monitoring and improving the energy efficiency of such steam distribution systems. To achieve this objective, Oak Ridge National Laboratory (ORNL) has recently conducted a pilot study to explore the deployment of a wireless sensor system for a real-time measurement-based energy efficiency optimization. For our campus, with particular focus on the 12-mile steam distribution network, we propose a holistic system-level approach to optimize energy delivery within the steam distribution system. In particular, we address the goal of saving energy in steam lines by monitoring and acting on leaking steam valves/traps. Our approach is through wireless sensor system integration and real-time monitoring system coupled with diagnostic algorithm.

We make assessments on the real-time status of the steam distribution system by mounting acoustic sensors on or near the steam pipes/traps/valves and capturing acoustic operating signatures 
fed through the algorithm to help diagnose the health of the steam system. Our assessments are based on real-time acoustic algorithms operating on the wireless sensor measurements. We describe a spectral-based energy signature approach* acting on the acoustic sensor data to classify the steam traps/valves status and estimate the steam flows in the pipes. This approach can be employed as the diagnostic engine for monitoring steam trap/flow health. The analysis based on estimated steam flow and steam trap status helps generate alerts that are presented as an interactive overlay within a webbased Google Earth geographic platform. The visualization of such alerts enables operators and maintenance personnel to take remedial action. Such an approach is expected to save as much as $\$ 675,000$ per year on the 12 -mile long steam distribution system at ORNL. ${ }^{2}$

We have organized the report to showcase our demonstration as an instantiation of a platform that extends implementation to include newer modalities in managing water flow, sewage, energy consumption that is reproducible in other sites such as in production lines of manufacturing companies. In Sect. 2, we present the overall system architecture, which includes the backhaul RF network for linking buildings, the steam wireless sensor network, link planning, system design and data flow. In Sect. 3, the spectral-based energy signature approach acting on the acoustic vibration sensor data is demonstrated. Experimental results demonstrating the extracted signatures for steam trap status classification and steam flow estimation are presented in Sect. 4 and Sect. 5, respectively. In Sect. 6, data visualization using a web-based Google Earth geographic platform is presented. We summarize our work and conclude with future directions in Sect. 7.

*The concept and original algorithm was developed in 1985 by Dr. Allgood and restructured in 2001 for use in a steam trap/energy computation environment. The algorithm was updated by Dr. Olama and Dr. Allgood for use in the particular environment. 


\section{COMMUNICATION SYSTEM ARCHITECTURE AND INTEGRATION}

The wireless communication system architecture is briefly described in this section. To accomplish measurement and other specific tasks associated with energy optimization, ORNL invoked an experimental architecture based on a suite of wireless sensors. Figure 1 describes the system architecture. We began with the construction of a secure wireless backbone providing connectivity for different wireless sensors mounted on pipes and valves spread across the campus. We also considered the future need to accommodate different modalities of sensors (such as temperature, pressure and flow) from different vendors. Different modalities and different vendors implied that we had to acquire data from sensors that may communicate using a diverse array of protocols such as MODBUS, ${ }^{3}$ OPC, ${ }^{4}$ and TCP/IP. ${ }^{5}$ Our solution to accommodate a variety of sensors and modalities was through the implementation of a middleware layer that understands different protocols. The middleware layer abstracts the vendor-specific protocols and interfaces to the operator. This solution ensures that when we have to add more sensors that adhere to newer/advanced communication protocols, the update to the wireless system only happens at the middleware layer. Another functionality of the middleware is to transform the data into a canonical form for archival in databases. The analysis and visualization components that we present in the later Sections of this report retrieve data from these databases.

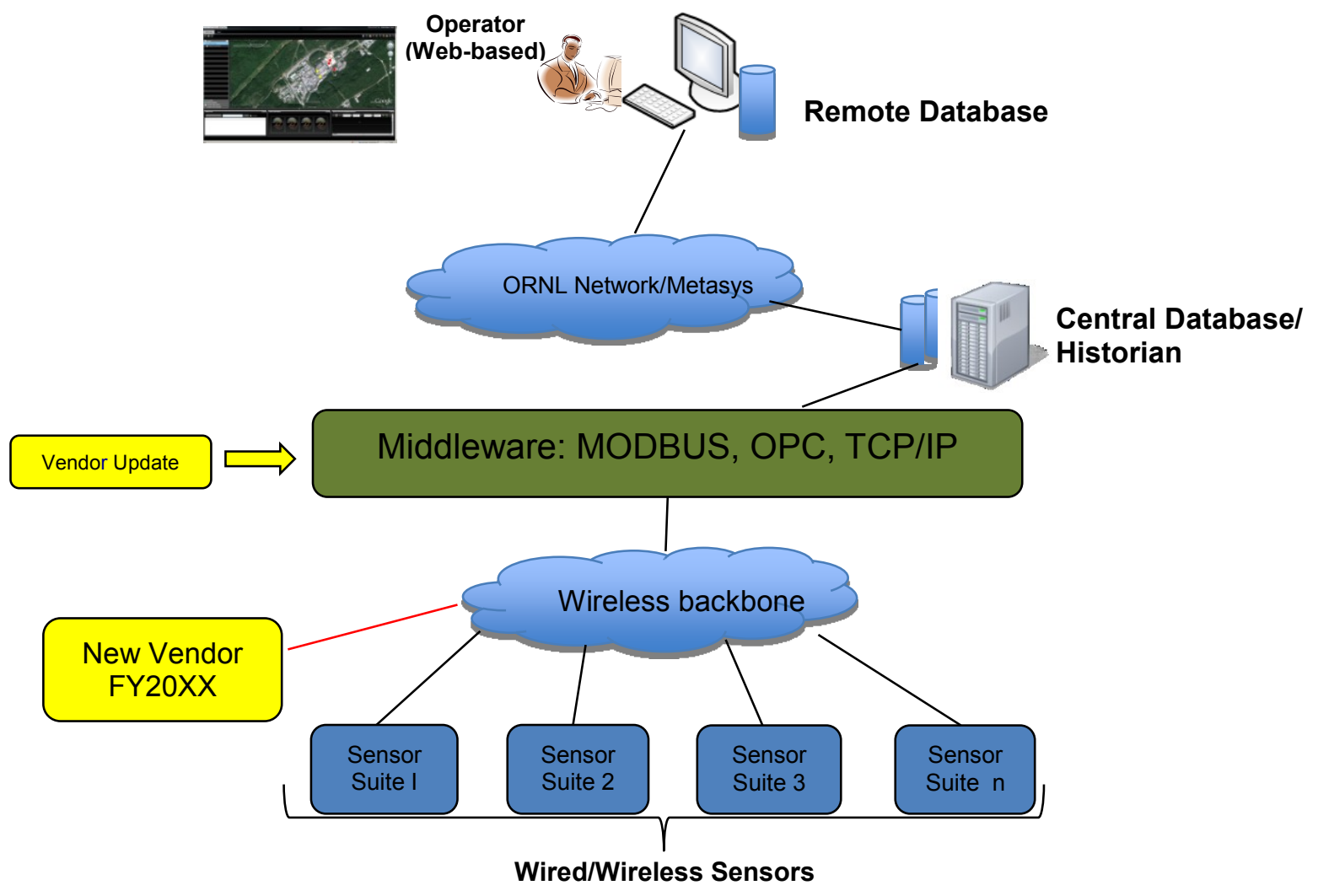

Fig. 1. Data-flow architecture of the ORNL wireless energy monitoring system. 
Plan view for the wireless infrastructure created for the pilot project at ORNL is illustrated in Fig. 2. There is a central antenna station that houses four sectored antennas to cover different areas of the steam lines along and remote antenna stations for local sensor data aggregation. Extensive consideration was given to cyber-security and site radio-frequency safety requirements. This pilot implementation used the industrial, scientific and medical (ISM) band at $5.8 \mathrm{GHz}$ to minimize impacts on the existing Wi-Fi infrastructure. Experiments were conducted to test signal strength and network availability across campus to ensure reliable coverage of the wireless infrastructure. The installed communication hardware enables sensor data flows to a centralized location. The centralized server processes the data to identify steam trap status and also estimate flow-rates within the distribution system. We describe the energy signature algorithm used for estimating steam trap/flow status in the following section.

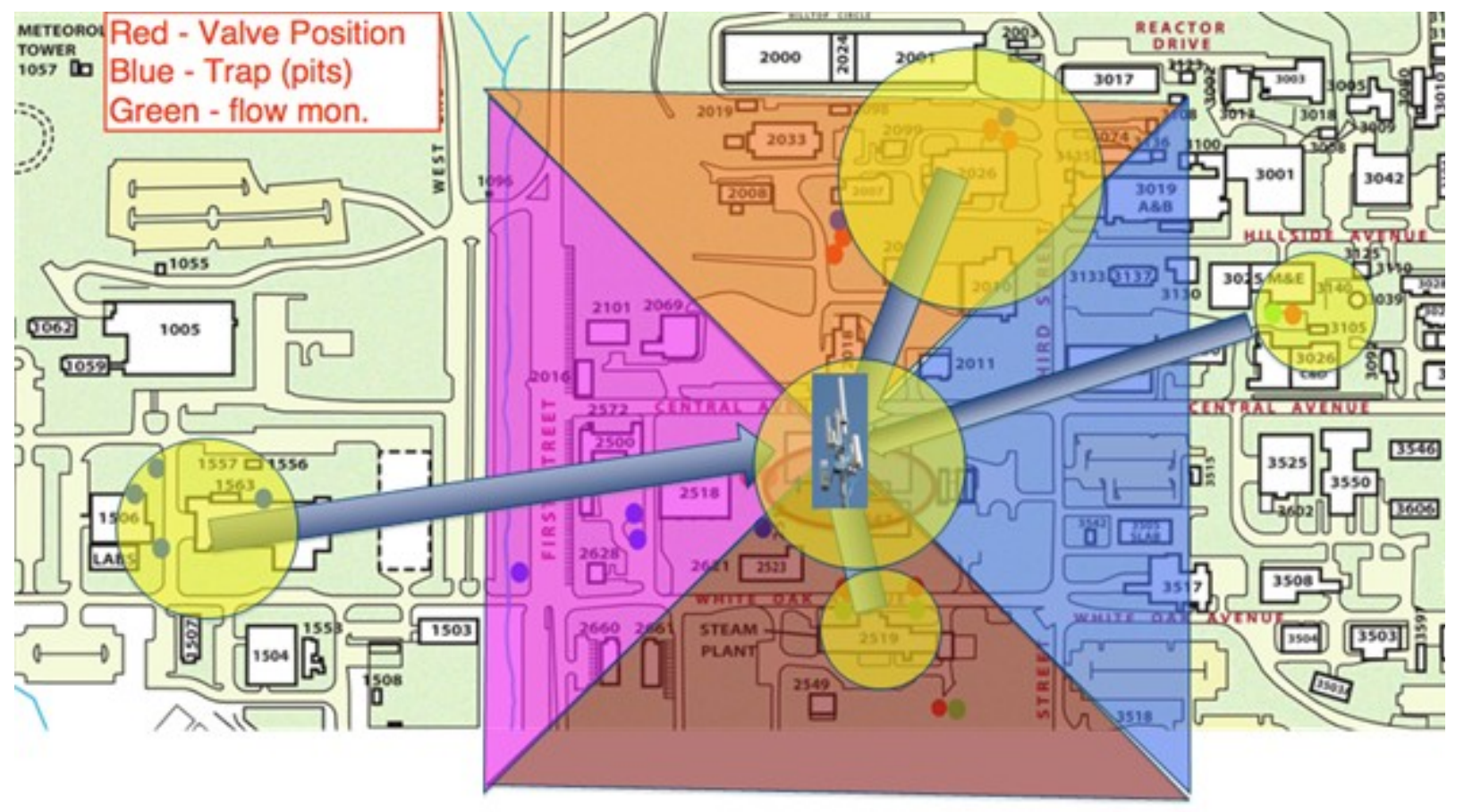

Fig. 2. Plan view of ORNL's campus showing the locations of the valves and steam traps in addition to antenna location and coverage. 


\section{THE PROPOSED ENERGY SIGNATURE SCHEME}

In this section, we develop a diagnostic capability that helps in assessing the operational health of a steam distribution system. In the event of a failure, we would like to identify the non-operational state with sufficient confidence to enable maintenance decisions to be enforced. This would not only reduce energy losses but also maintenance costs while increasing availability of the system. To achieve this task, acoustic sensors were strategically placed on or near steam pipes/traps/valves to capture operating signatures that help diagnose the health of the steam system.

In the following paragraph, we formulate a spectral-based approach, which can be employed as the diagnostic engine for monitoring a steam distribution system health. The proposed formulation performs energy signature analysis on the time-domain vibration measurement data received from the acoustic sensors to identify/estimate the various states of their associated steam traps/flows. The first step involves transforming the discrete-time vibration signal, $x(n)$, to the frequency-domain by using the short-time Fourier transform (STFT) as ${ }^{6}$

$$
X(k, f)=\sum_{-\infty}^{\infty} x(n) \omega(n-k) e^{-j 2 \pi f n}
$$

where $\omega(n)$ is the window function. In our analysis in this report a Hamming window is considered. Then the spectrogram, or the time-frequency power spectral density (PSD), ${ }^{7}$ can be estimated by computing the squared magnitude of the STFT of the signal $x(n)$ as ${ }^{8}$

$$
\operatorname{PSD}(k, f)=|X(k, f)|^{2}
$$

Finally, the time-frequency PSD is used to compute energy signatures, $\beta_{i, k}$, as described by the following equations:

$$
\begin{gathered}
\beta_{i, k}=\int_{f_{i_{\text {lower }}}}^{f_{i_{\text {upper }}} \operatorname{PSD}(f, k) d f} \\
\beta_{k}=\sqrt{\sum_{i=1}^{I} \beta_{i, k}^{2}} \\
\beta_{\text {Total }}(t)=\sum_{k=0}^{t} \beta_{k}
\end{gathered}
$$

where $f_{i}$ 's represent the frequency intervals that capture the spectral features, $0 \leq i \leq I, 0 \leq k \leq K$, $0 \leq t \leq K, I$ is the total number of spectral feature sets, and $K$ is the total number of discrete-time epochs.

To identify the $\beta_{i, k}$ of interests and their associated elements, that include $f_{i}$ 's, a complete operational analysis over all ranges of steam trap operations and operational states must be conducted. Such an effort could include a statistical design of experiments approach when all experimental factors and levels cannot be controlled due to operational restrictions. Most steam distribution systems are designed for operator-based flow control. Therefore, for the experiments presented in this report, we assumed that measurements of all operational states of the distribution system are available. Once all the data is collected, each operational level/state combination is analyzed. We then extract spectral features that will be used in a sensitivity analysis to identify a compact set of $\beta_{i, k}$ to use in the diagnostic algorithm. Given the $\beta_{i, k}$ in Eq. (3), a vector set of Eqs. (4)-(5) are calculated representing each specific $\beta_{k}$ as well as a $\beta_{\text {Total }}$ calculation.

We demonstrate the viability of the energy signature scheme in assessing steam trap status and estimating steam flow rates in Sect. 4 and Sect. 5, respectively. 


\section{EXTRACTING SIGNATURES FOR STEAM TRAP STATUS CLASSIFICATION}

In this section, we demonstrate the effectiveness of the energy signature approach discussed in the previous section in assessing the operational health of a steam trap. Figure 3 shows the timefrequency PSDs, or spectrograms, conducted for a class of steam traps under similar operating conditions and three operational states: (i) New - 125 psi / 20 lbs; (ii) Rupture - 125 psi / 20 lbs; and (iii) Over Press $-125 \mathrm{psi} / 20$ lbs. These results were generated using real accelerometer data sampled at $20 \mathrm{KHz}$, and each segment (time epoch) in the spectrogram computations is windowed with a Hamming window of length 4096 samples with 50 samples overlap between segments.

The corresponding overall $\beta_{\text {Total }}$ for the three operational states described in Fig. 3 as a function of discrete time are shown in Fig. 4 . In this figure, $\beta_{\text {Total }}$ is computed by applying Eqs. (3)-(5). Two things are evident from Fig. 4. First, the final integrated $\beta_{\text {Total }}$ value for each operational state/condition pairing has sufficient separation in the $\beta_{\text {Total }}$ space to use as a diagnostic/performance indicator. Second, the dynamics exhibited by each operational state/condition pairing $\beta_{\text {Total }}$ over the discrete time of interest is sufficiently different that it could be used as additional support for identifying the steam traps states. Given the characteristics and values of energy signatures, $\beta_{\text {Total }}$ in Fig. 4, a classification algorithm can be constructed to identify the state of the steam trap under consideration. Figure 5 shows the final $\beta_{\text {Total }}$ for data sets collected at the input and output of a steam trap that is considered in good state. Notice that the final $\beta_{\text {Total }}$ does not change much when changing the location of the acoustic sensor from the input to the output of the steam trap. Therefore, we conclude that the proposed steam trap health monitoring algorithm is not sensitive to the location of the sensors. This spectral-based approach helps generate alerts based on the estimated signatures from acoustic data.

Another parameter required for ensuring efficient steam delivery is the flow rate. Although a simple solution would be to include flow meters into the steam distribution system, off-the-shelf flow meter installation requires at least a partial stoppage of the distribution line for sensor installation. In the next section, a nonintrusive approach to estimation of steam flow from accelerometer data is discussed. Such an approach could reduce the hardware and installation costs required for new flow meters. It also avoids the risk of having to completely or partially sever steam supplies to the industrial operations. 


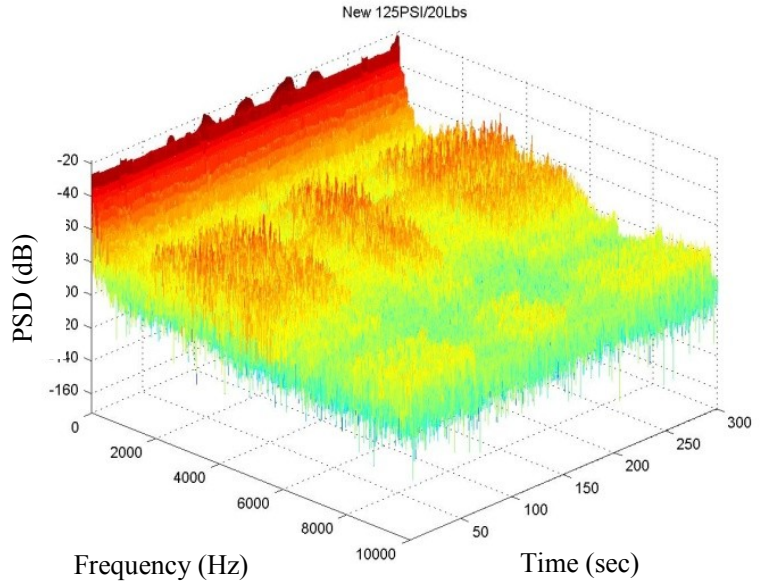

(a)

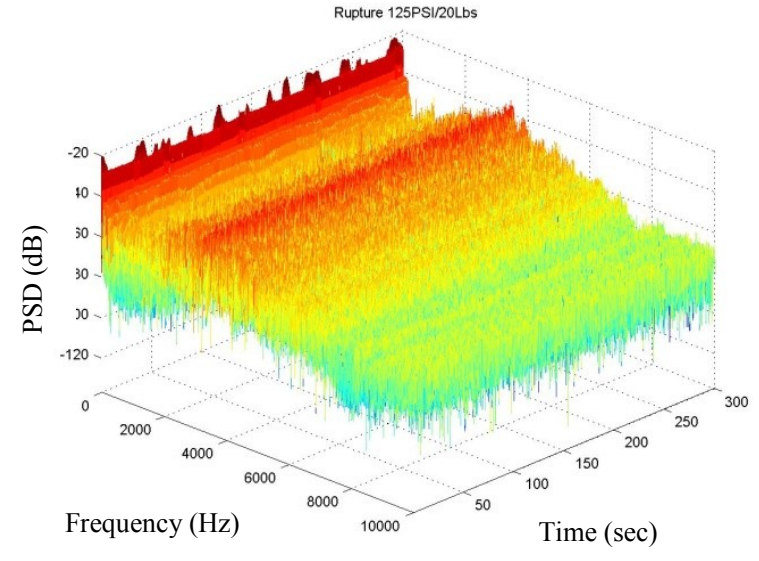

(b)

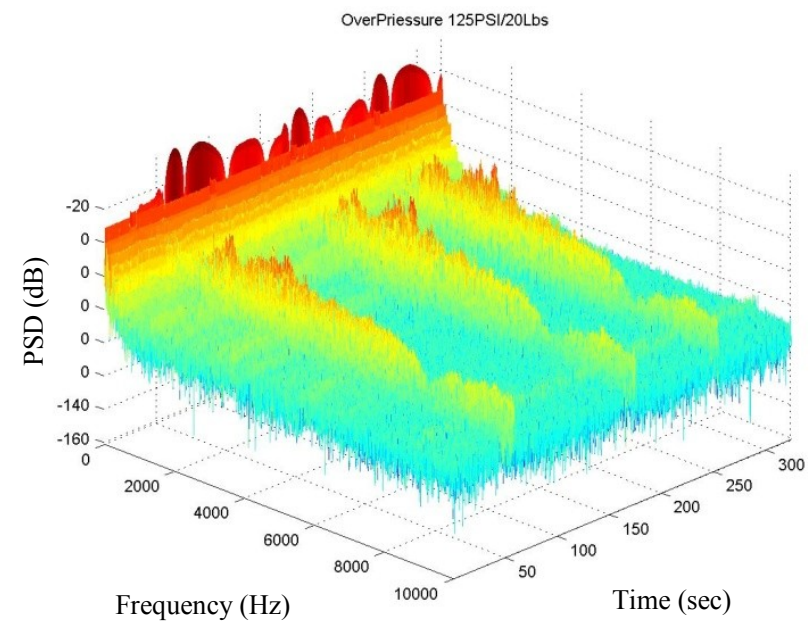

(c)

Fig. 3. Time-frequency PSDs of a single class of steam trap types showing similar operational ranges and varying states: (a) Signatures for New-125 psi / 20 lbs; (b) Signatures for Rupture -125 psi / 20 lbs; and (c) Signatures for Over Press-125 psi / 20 lbs. 


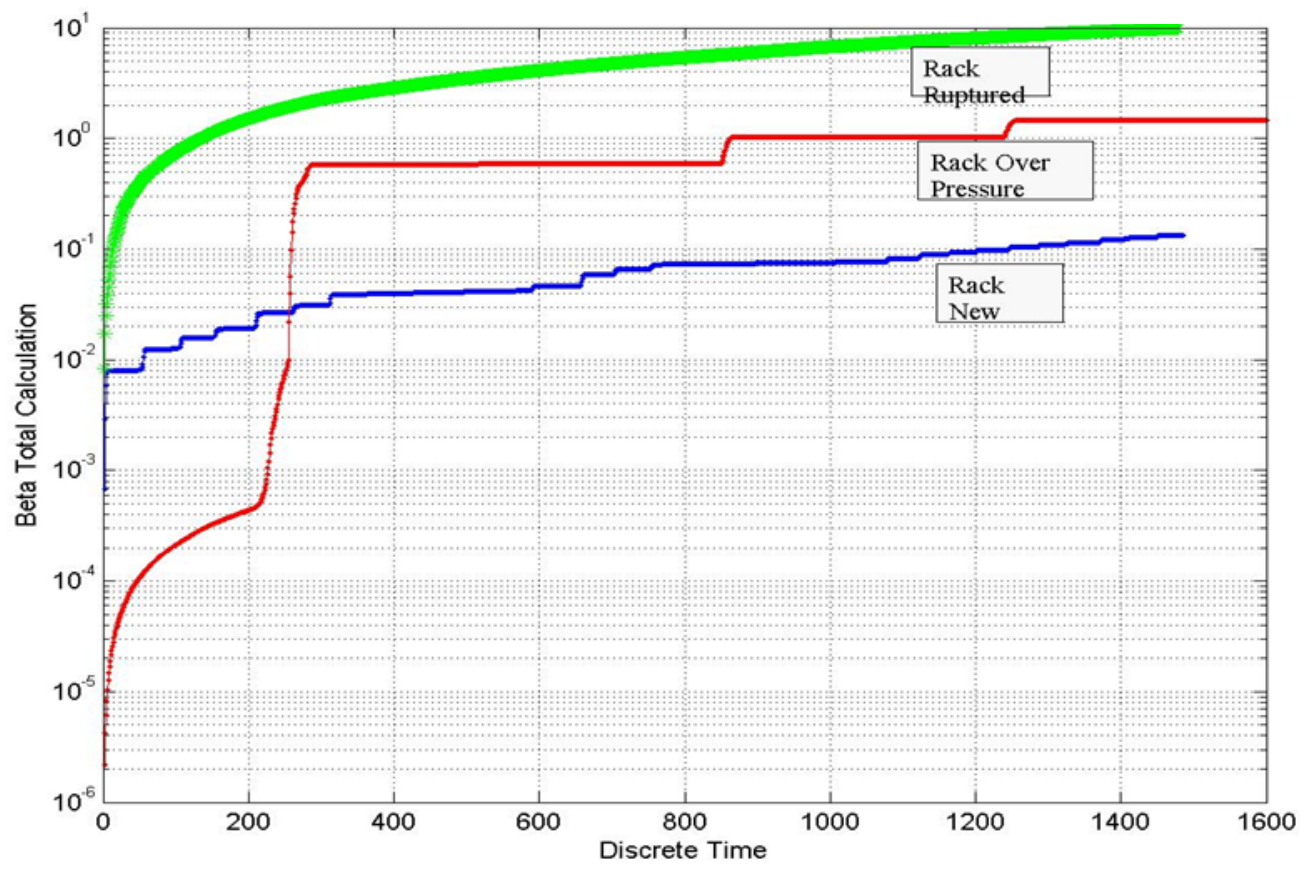

Fig. 4. Energy signatures for the three states and health under similar operational conditions (125 psi / 20 lbs).

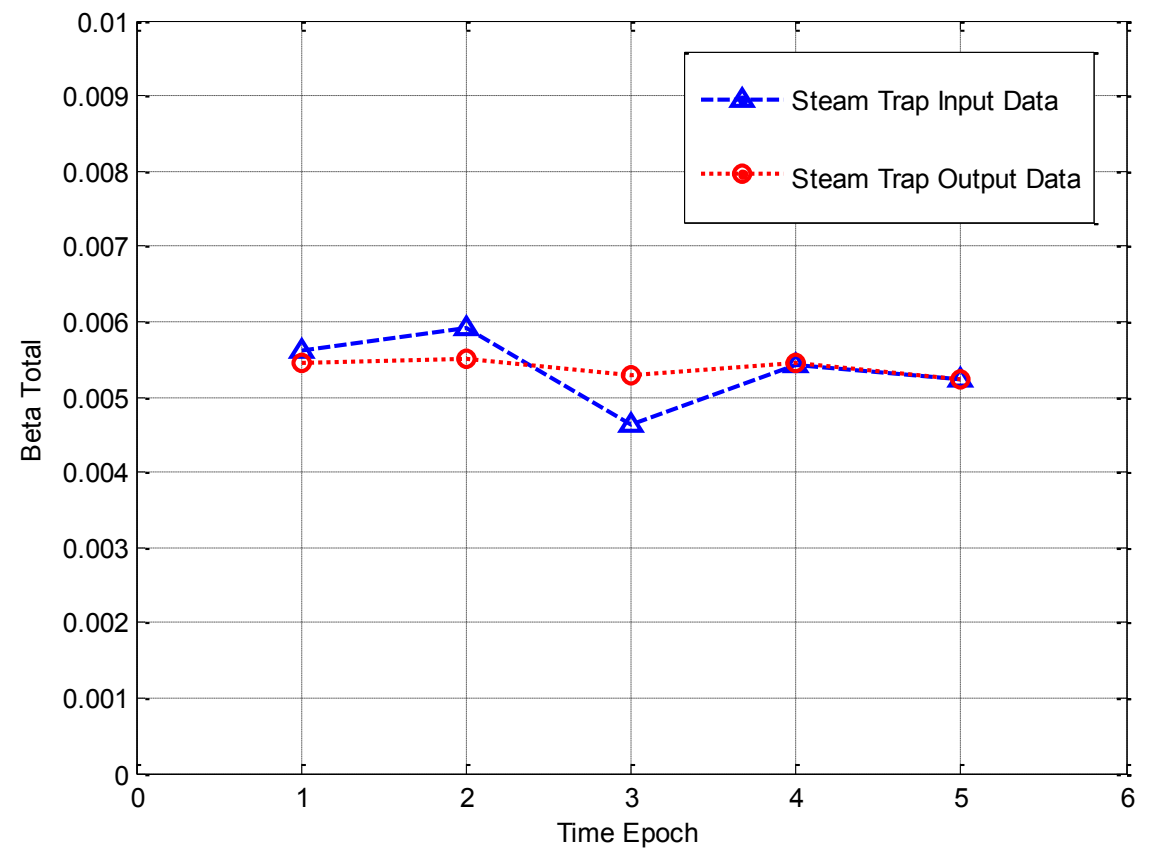

Fig. 5. Energy signature values computed on data acquired from the input and output sections of a steam trap. The graph shows that the location of the accelerometer does not drastically influence the prediction of the steam trap status. 


\section{EXTRACTING SIGNATURES FOR STEAM FLOW ESTIMATION}

In this section, we employ the energy signature scheme described in Sect. 3 for estimating steam flow rates from vibration (acoustic) measurements. Before we present the numerical results of the energy signature scheme, we analyze some statistical properties of the collected vibration measurement data. The collected vibration data were sampled at $1 \mathrm{KHz}$ and over fixed flow rate periods. Seven different steam flow rates data are considered in our analysis and we randomly choose 4096 samples at each flow rate. Figure 6 demonstrates box-and-whisker plot $^{9}$ for ten data sets collected at a flow rate of $2390 \mathrm{~L} / \mathrm{Min}$ and at different times over a particular week (and at different time instances throughout the day). Note that lower and upper lines of the "box" are the $25^{\text {th }}$ and $75^{\text {th }}$ percentiles of the sample, respectively, the circle in the middle of the box is the sample median, and the whiskers are lines extending from each end of the box to show the extent of the rest of the data. It can be observed from Fig. 6 that the vibration measurement data are essentially stationary since their statistics are almost fixed at different time instances.

The relationship between the overall $\beta_{\text {Total }}$ resulting from performing the energy signature scheme and steam flow rate is shown in Fig. 7. As seen, the overall $\beta_{\text {Total }}$ is increasing with increasing flow rate from $2.68 \times 10^{-5}$ to $1.68 \times 10^{-4}$. The solid line represents a second-order least squared error fit to the data. The equation for the curve fit can be expressed as follows:

$$
\beta_{\text {Total }}(r)=a r^{2}+b r+c
$$

where $a=8.4901 \times 10^{-10}, b=-3.7231 \times 10^{-6}, c=0.0041$, and $r$ is the steam flow rate in L/Min. Figure 7 clearly shows that there is a strong correlation between the energy signature parameter, $\beta_{\text {Total }}$, and flow rates and they can be fit by a quadratic equation. This agrees with the main conclusion of Evans et al., ${ }^{10}$ which states that sound and vibration signals recorded by the accelerometer mounted to the surface of a pipe has a strong (deterministic) relationship with the flow rate. Based on the real-time vibrations as recorded by the accelerometers, we can then estimate the steam flow rate by computing the overall $\beta_{\text {Total }}$ using Eqs. (1)-(5) and solving for flow-rate estimates using Eq. (6).

Now, in order to assess how confident the flow rate estimate is, we conducted sensitivity analysis for energy signature values, $\beta_{\text {Total }}$, computed on vibration data at different steam flow rates. Figure 8 demonstrates box-and-whisker plot for 10 data sets collected at each flow rate over different time instances. Note that the notches, in a notched box plot, represent a robust estimate of the uncertainty about the medians for box-to-box comparison. Boxes whose notches do not overlap indicate that the medians of the two groups differ at the 5\% significance level. It can be observed from Fig. 8 that the boxes do not overlap and the separation between boxes increase with increasing flow rate. This indicates that the energy signature scheme has sufficient sensitivity to provide trust in the estimated steam flow rate, and the performance improves at high flow rates.

Thus far, we presented a scheme to estimate steam traps/valves status and steam flow rates at control point within the distribution system. In the following section, we present the estimated status and flow rates in addition to raw sensor data as feeds on a web-based interface. 


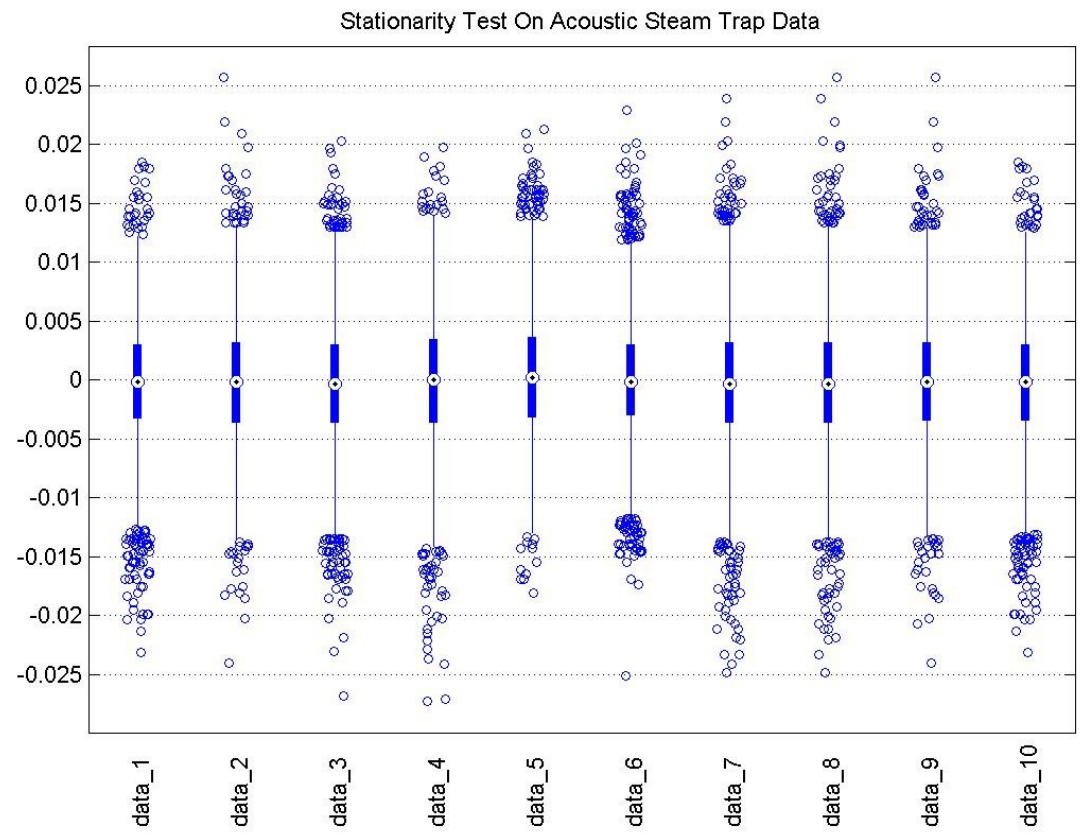

Fig. 6. Box-and-whisker plot for 10 data sets collected at a fixed steam flow rate of $2390 \mathrm{~L} / \mathrm{Min}$ and at different time instances over a particular week. The graph shows that the vibration measurement data are essentially stationary.

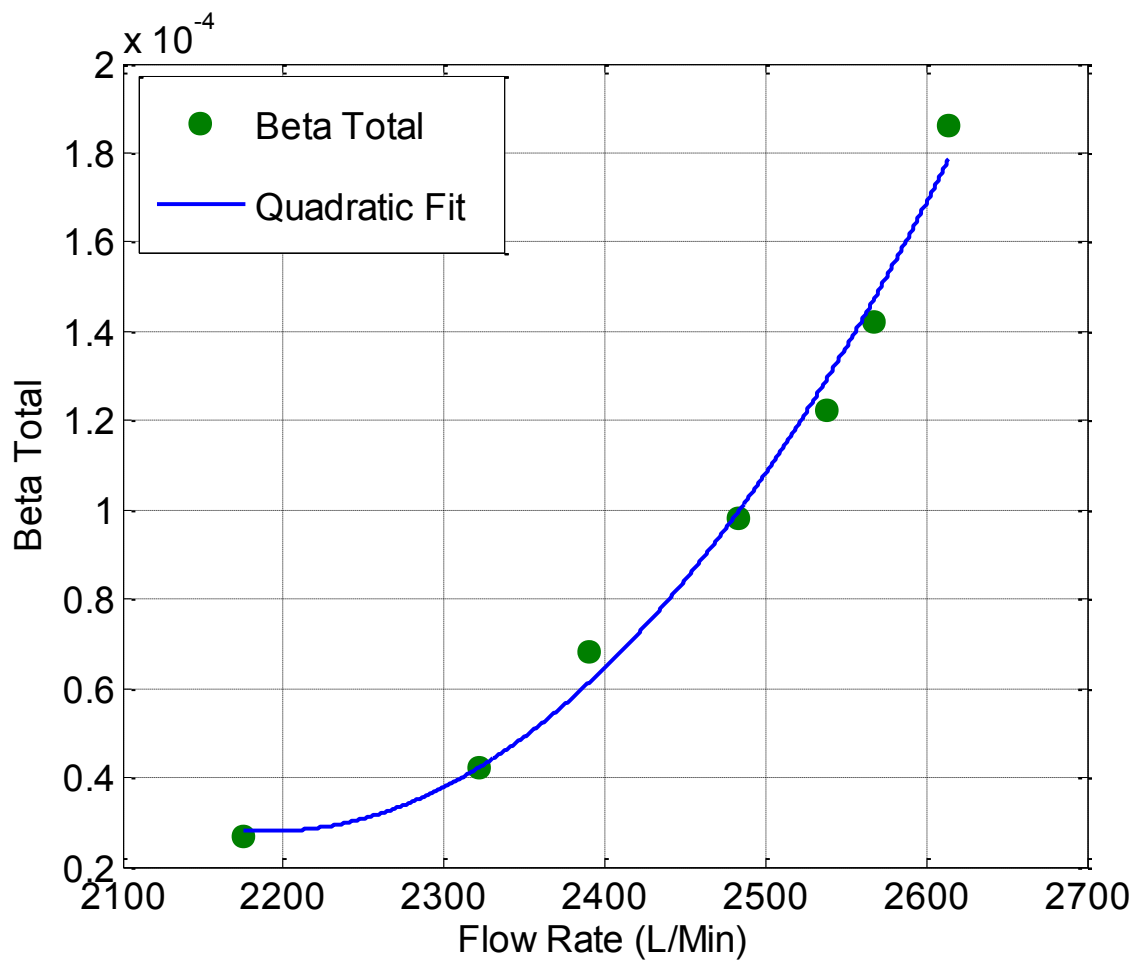

Fig. 7. Energy signature values computed on vibration data at different steam flow rates. The graph shows that the energy signature parameter, $\beta_{\text {Total }}$, is quadratically proportional to the steam flow rate. 


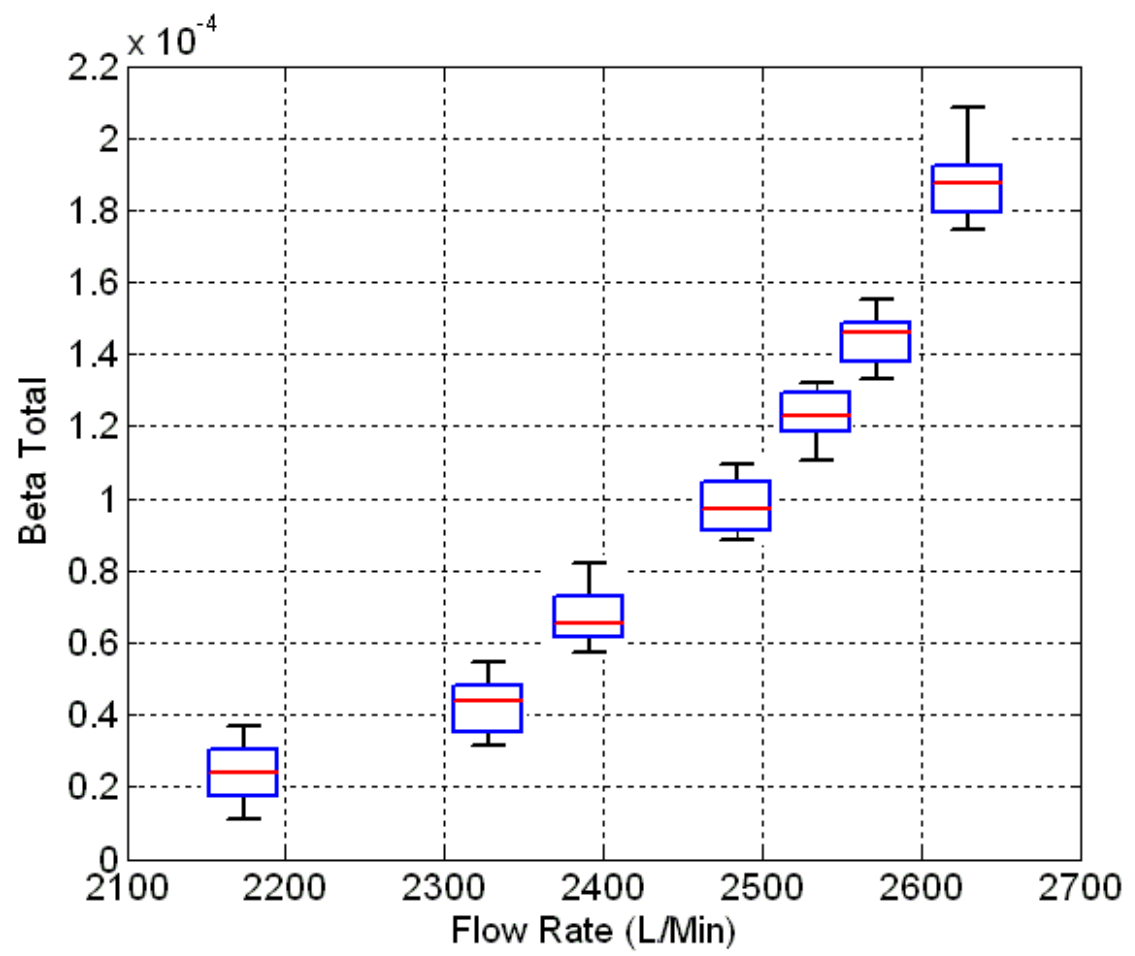

Fig. 8. Sensitivity analysis for energy signature values computed on vibration data at different steam flow rates. The graph shows that the energy signature scheme has sufficient sensitivity to provide trust in the estimated flow rate. 


\section{VISUALIZATION}

As introduced earlier in Fig. 1, the wireless sensor data along with the computed estimates of steam trap status and flow rates are brought to a centralized server and archived into a database that can be retrieved upon user request. The data is then made available to operators and maintenance personnel using a browser-based delivery mechanism ${ }^{11}$ as shown in Fig. 9. The browser based delivery is mobile-phone friendly and very useful for field technicians. Leveraging geo-tagged locations of the steam valves, traps and pipes in the steam distribution system, we are able to deliver sensor data as a Google-Earth ${ }^{12}$ overlay.

The green circles on the map in Fig. 9 indicate steam valves operating under normal conditions. The red circles indicate potential faults. The radius of the red circles encode the duration for which the potential fault has been identified. Potential faults could be traps failing closed, traps failing open or traps leaking. We use different colors to represent different failure modes. The ability to visualize campus-wide sensor data enables personnel to plan a suitable course of action while maintaining the requirements needed for the industrial operations. The insets below the map, show a table of the most recent measurement values at each of the sensors (bottom far right), measurements of temperature, pressure, flow and battery life as dashboard at a particular building (center) and the list of potential areas of concern within the distribution system (bottom far left). The Google-Earth interface provides an intuitive and interactive mechanism for operators and field technicians to understand the status of the steam distribution system - both during a fault and after a fault has been repaired. We have also implemented the ability to query the history of the steam distribution system from this interface. The slider bar on the top left of the map allows the user to see temporal snapshots of the distribution system. The user-interface design can accommodate a variety of sensors including ones planned in the future for waste management and energy consumption.

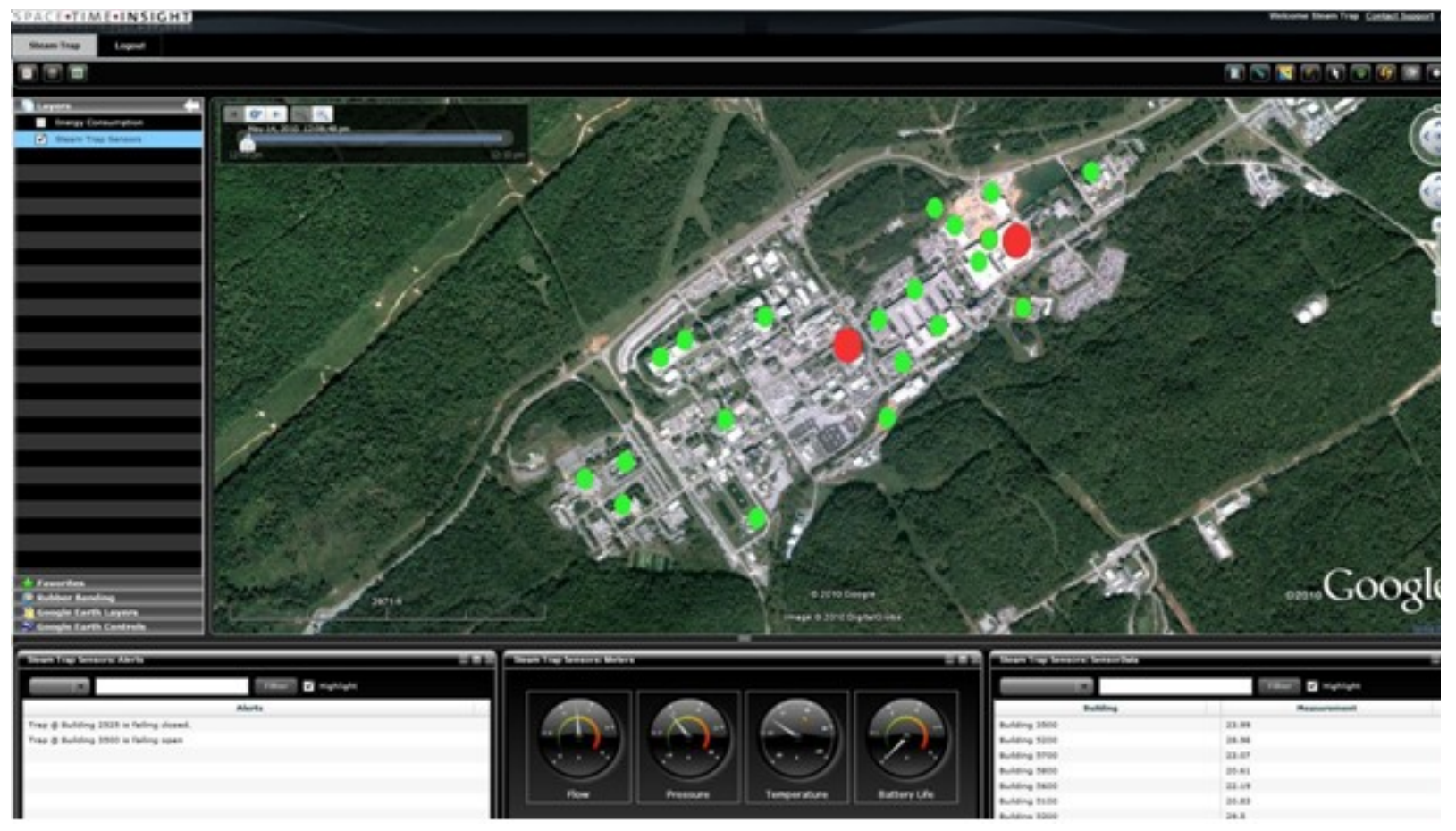

Fig. 9. The situational awareness interface used to visualize sensor data from the wireless sensors along with status alerts. 


\section{CONCLUSIONS}

Based on off-the-shelf inexpensive technologies, we proposed the development of a measurement-based energy efficiency optimization framework for a steam distribution system. A spectral-based energy signature scheme acting on real-time acoustic accelerometer sensor measurements acquired over a wireless network was described. The scheme identified steam traps status and estimated steam flows in the system. Experimental results show that the acoustic signature approach has the potential to identify different steam trap health status and it has sufficient sensitivity to estimate steam flow rate. Moreover, results indicate a nearly quadratic relationship over the test region between the overall energy signature factor and flow rate in the pipe. The proposed scheme operates on accelerometer measurements collected from acoustic sensors mounted outside steam pipes/traps, and therefore it is a nonintrusive approach and an alternative for flow meters that are mounted inside the steam pipes. Future work is to study the effect of pipe material/diameter and different steam trap types on the performance of the acoustic signature scheme.

All readings, charts, and steam traps status are visualized in an interactive overlay within a webbased Google Earth geographic platform. We envision integrating several sensor sources into a unified site-wide monitoring framework. Leveraging the wireless infrastructure, we believe our demonstration serves as an instantiation of a platform that extends implementation to include newer modalities in managing water flow, sewage and energy consumption that is reproducible in other sites such as in production lines of manufacturing companies. 


\section{REFERENCES}

1. T. Collins, S. A. Parker, and D. Brown, Steam Trap Performance Assessment: Advanced Technologies for Evaluating the Performance of Steam Traps, U.S. Department of Energy, Pacific Northwest National Laboratory, 2004.

2. R\&D Daily, Taking Steam Out of Wasted Energy, R\&D Magazine, Oct. 6, 2010.

3. B. Drury, Control Techniques Drives and Controls Handbook, Institution of Engineering and Technology, $2^{\text {nd }}$ Edition, 2009.

4. About OPC - OPen Connectivity through Open Standards, Kepware Technologies, 2009.

5. D. E. Comer, Internetworking with TCP/IP: Principles, Protocols, and Architecture, Prentice Hall, $5^{\text {th }}$ Edition, 2006.

6. R. N. Bracewell, The Fourier Transform and Its Applications, $3^{\text {rd }}$ Edition, Boston: McGrawHill, 2000.

7. A. V. Oppenheim and R. W. Schafer, Discrete-Time Signal Processing, Prentice-Hall, Englewood Cliffs, NJ, 713-718, 1989.

8. S. Haykin, Advances in Spectrum Analysis and Array Processing, vol. 1, Prentice-Hall, 1991.

9. R. McGill, J. W. Tukey, and W. A. Larsen, Variations of Boxplots, The American Statistician, vol. 32, 12-16, 1978.

10. R. P. Evans, J. D. Blotter, and A. G. Stephens, Flow Rate Measurements Using FlowInduced Pipe Vibration, Journal of Fluids Engineering, vol. 126, no. 2, 280-285, 2004.

11. Accessed on Oct 1, 2011, www.spacetimeinsight.com

12. Accessed on Oct 1, 2011, www.earth.google.com 